\title{
Successfully Treated Isolated Posterior Spinal Artery Aneurysm Causing Intracranial Subarachnoid Hemorrhage: Case Report
}

\author{
Yoshinobu HORIO, ${ }^{1}$ Toshiro KATSUTA, ${ }^{1}$ Kazuhiro SAMURA, ${ }^{1}$ Naoki WAKUTA, ${ }^{1}$ \\ Kenji FuKudA, ${ }^{1}$ Toshio HigAsHI, ${ }^{1}$ and Tooru INOUE ${ }^{1}$ \\ ${ }^{1}$ Department of Neurosurgery, Faculty of Medicine, Fukuoka University, Fukuoka
}

\begin{abstract}
There are very few published reports of rupture of an isolated posterior spinal artery (PSA) aneurysm, and consequently the optimal therapeutic strategy is debatable. An 84-year-old man presented with sudden onset of restlessness and disorientation. Neuroradiological imaging showed an intracranial subarachnoid hemorrhage (SAH) with no visible intracranial vascular lesion. Spinal magnetic resonance imaging (MRI) detected a localized subarachnoid hematoma at Th10-11. Both contrast-enhanced spinal computed tomography and enhanced MRI and magnetic resonance angiography revealed an area of enhancement within the hematoma. Superselective angiography of the left Th12 intercostal artery demonstrated a faintly enhanced spot in the venous phase. Thirteen days after the onset of symptoms, a small fusiform aneurysm situated on the radiculopial artery was resected. The patient's postoperative course was uneventful and he was eventually discharged in an ambulatory condition. To our knowledge, this 84-year-old man is the oldest reported case of surgical management of a ruptured isolated PSA aneurysm. This case illustrates both the validity and efficacy of this therapeutic approach.
\end{abstract}

Key words: posterior spinal artery, radiculopial artery, isolated aneurysm, spinal aneurysm, spinal subarachnoid hemorrhage

\section{Introduction}

Subarachnoid hemorrhage (SAH) of spinal origin is rare. Approximately, only 1\% of cases of intracranial SAH are related to spinal pathologies. ${ }^{11}$ The most common cause is spinal cord arteriovenous malformations, followed by spinal tumors such as ependymomas and neurinomas. ${ }^{2)}$ Rupture of spinal artery aneurysms is rare and in most cases associated with vascular anomalies that cause hemodynamic stress such as spinal arteriovenous malformations or coarctation of the aorta. ${ }^{3)}$ Connective tissue disease or vasculitis of infectious or autoimmune etiology are thought to be other causes. ${ }^{3)}$ Isolated spinal aneurysms that are not associated with vascular malformations or above-mentioned predisposing factors are extremely rare, and majority of them are located at the anterior spinal artery. ${ }^{4)}$ We here report a case of SAH caused by rupture of an isolated posterior spinal artery (PSA) aneurysm that was successfully treated surgically. Because there are few reports of isolated PSA aneurysms, the optimal therapeutic strategy is debatable.

Received August 8, 2015; Accepted October 6, 2015

\section{Case Report}

An 84-year-old man who was being treated for right thalamic infarction in another hospital presented with restlessness and disorientation of sudden onset. Computed tomography (CT) demonstrated an intracranial SAH (Fig. 1A); however, magnetic resonance angiography (MRA) failed to identify an intracranial aneurysm. Spinal magnetic resonance imaging (MRI) detected a localized subarachnoid hematoma at Th10-11 (Fig. 1B). He was transferred to our hospital on the same day for further examination and treatment.

On admission, he was disorientated and had left hemiplegia and sensory disturbance, which were attributed to the known right thalamic infarction. He had no symptoms or signs of spinal cord or root lesions. Computed tomography angiography of the head showed no aneurysms or vascular malformations. Contrast-enhanced spinal CT revealed an area of enhancement on the left dorsal aspect of spinal cord at Th10-11 (Fig. 2A). Enhanced MRI and MRA 5 days after the onset of symptoms also showed enhancement in the center of the localized hematoma (Fig. 2B). Nine days after onset, digital subtraction angiography with superselective angiography of the left 


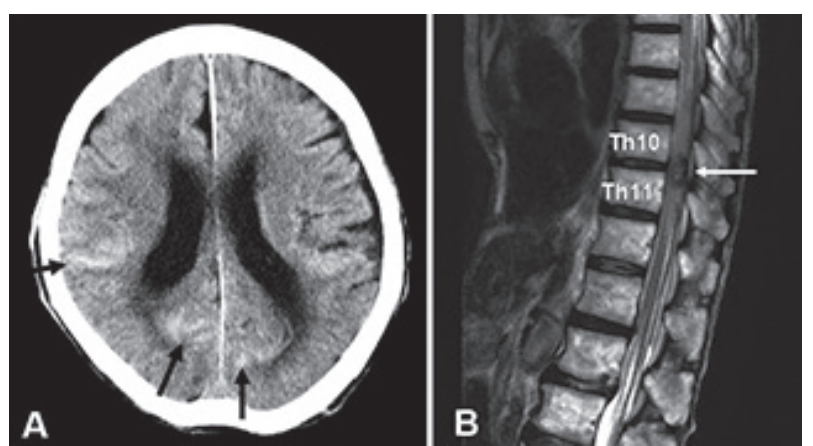

Fig. 1 Initial neuroradiological imagings. A: Brain computed tomography showing intracranial subarachnoid hemorrhage (arrows). B: Spinal MRI ( $\mathrm{T}_{2}$-weighted image). The hypointensity lesion at Th10-11 (arrow) suggests a localized subarachnoid hematoma.
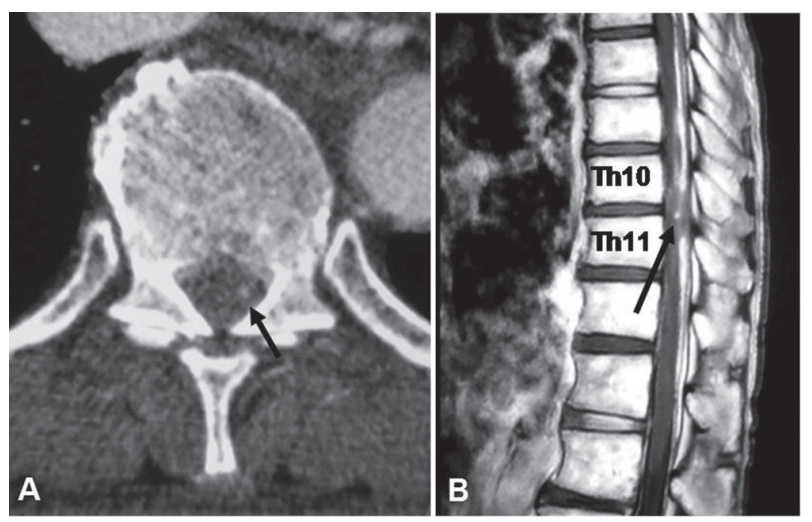

Fig. 2 Contrast-enhanced computed tomography (day 1: A) and spinal magnetic resonance imaging (day 5: B). A: There is an area of enhancement on left dorsal aspect of the spinal cord at Th10-11 (arrow). B: Contrast-enhanced $\mathrm{T}_{1}$-weighted image showing an area of enhancement within the hematoma (arrow).

Th12 intercostal artery demonstrated an area of faint enhancement in the venous phase. However, the connections with the surrounding vessels were unclear (Fig. 3).

The imaging findings were thus highly suggestive of SAH caused by spinal artery aneurysm. Dural arteriovenous fistula and cavernomas were among the different diagnosis at this time. To prevent rebleeding, surgery was performed 13 days after the onset.

A Th10-11 laminectomy was performed to approach the lesion. When the dura mater and arachnoid were opened, old yellowish hematoma was exposed on the left dorsal aspect of the spinal cord. Careful removal of the hematoma revealed a small fusiform aneurysm situated on the radiculopial artery, which ran along the dorsal root. Indocyanine green video-angiography failed to visualize the aneurysm, suggesting that the lumen was mostly thrombosed. The parent artery was coagulated and cut both distal and proximal to the aneurysm and the aneurysm resected en bloc (Fig. 4).

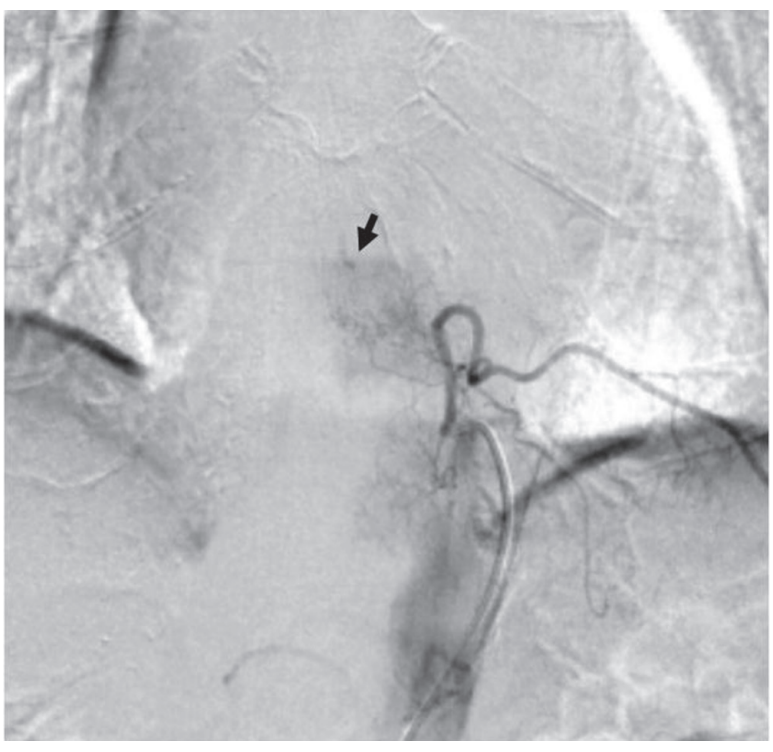

Fig. 3 Spinal digital subtraction angiography (day 9). Superselective angiographic image of the Th12 intercostal artery showing a faintly enhanced area in the venous phase (arrow). The connections with surrounding vessels are not clear.

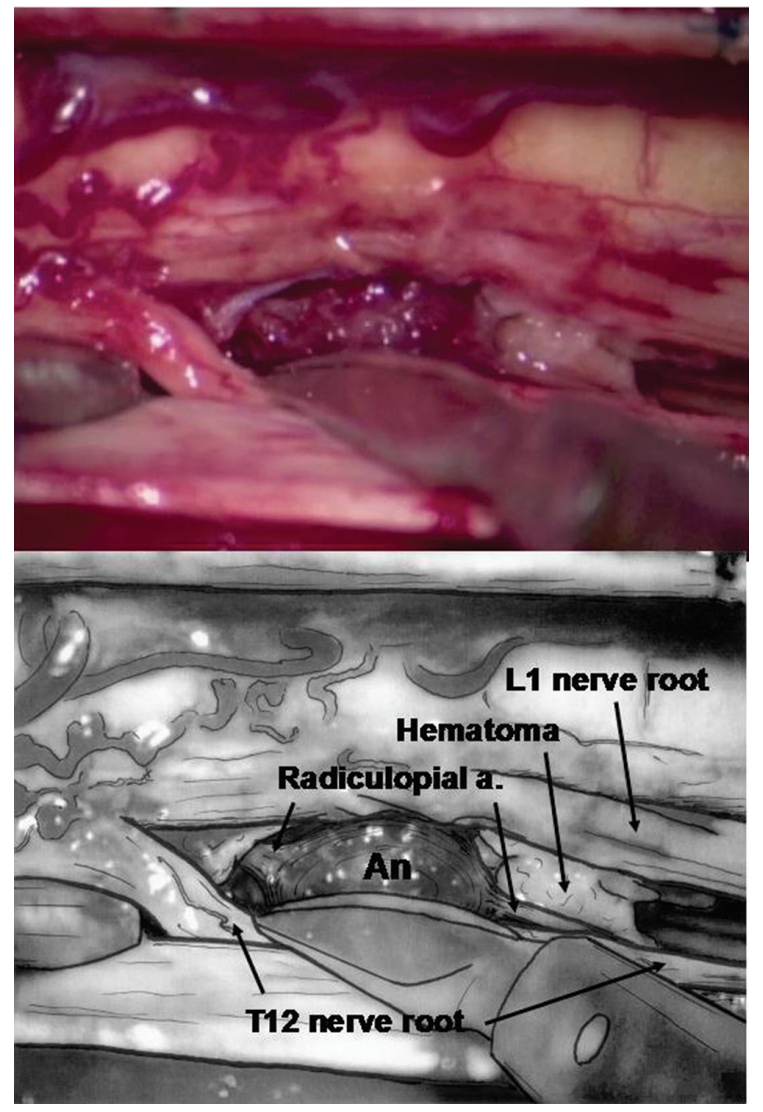

Fig. 4 Intraoperative image (day 13). After removal of the yellowish-white hematoma on the left dorsal aspect of the spinal cord, a fusiform aneurysm was detected on the radiculopial artery. An: aneurysm, Radiculopial a.: radiculopial artery. 
The patient's postoperative course was uneventful and rehabilitation was soon commenced. His activities of daily living gradually improved and he was transferred to another hospital for further rehabilitation, finally being discharged in an ambulatory condition 5 months after the onset of symptoms. Histopathological analysis revealed a disrupted elastic lamina in part and slightly organized thrombus in and around the aneurysmal wall.

\section{Discussion}

The ventral aspect of spinal cord is fed by the radiculomedullary artery, which is connected to the anterior spinal artery. The dorsal aspect is fed by the radiculopial artery, which has a communication with vasa corona, the latter mainly consisting of a pair of PSAs. ${ }^{5)}$ Our patient's SAH was caused by rupture of an isolated radiculopial artery aneurysm. Aneurysms of this artery are often reported as isolated PSA aneurysms. To our knowledge, this is the 21st reported isolated PSA aneurysm (Table 1).

Most of the isolated PSA aneurysms are considered to have been caused by dissection. ${ }^{6,7)}$ In our case, histopathological examination of the resected specimen failed to reveal a pseudolumen, probably because resection was performed 13 days after the onset of symptoms. However, its fusiform shape and location in a site unrelated to arterial branching were consistent with dissecting aneurysm.

Ruptured isolated PSA aneurysms have been treated by surgery, endovascular embolization, or conservatively. Because PSA aneurysms are located on the dorsolateral aspect of the spinal cord, they can easily be approached surgically. Most reported cases have therefore been managed surgically. ${ }^{1,2,6-15)}$ Endovascular embolization was performed in four of the reported cases. ${ }^{1,4,16,17)}$ However,

Table 1 Treatment of ruptured isolated posterior spinal artery aneurysms

\begin{tabular}{|c|c|c|c|c|c|}
\hline Author (year ) & Age/Sex & Level & Treatment & Outcome & $\begin{array}{c}\text { Timing of } \\
\text { intervention } \\
\text { or rebleed }\end{array}$ \\
\hline Henson \& Croft $(1956)^{19)}$ & $51 / \mathrm{M}$ & $\mathrm{C} 1$ & Conservative & Dead & 8 months rebleed \\
\hline Koçak et al. $(2006)^{20)}$ & $54 / F$ & $\mathrm{C} 2$ & Conservative (rebleed before $\mathrm{Tx}$ ) & Dead & $\begin{array}{l}\text { within } 24 \text { hours } \\
\text { rebleed }\end{array}$ \\
\hline Sato et al. (2012) ${ }^{18)}$ & $67 / F$ & T8 & Conservative & Good & - \\
\hline van Es et al. $(2013)^{10)}$ & $68 / \mathrm{M}$ & $\mathrm{T} 4$ & Conservative & Good & - \\
\hline Berlis et al. $(2005)^{2)}$ & $62 / \mathrm{F}$ & T5 & $\begin{array}{l}\text { Laminectomy (thrombosis } \\
\text { confirmed) }\end{array}$ & Good & 26 days \\
\hline Goto et al. $(1988)^{8)}$ & $53 / \mathrm{M}$ & $\mathrm{C} 2$ & Resection & Good & 2 days \\
\hline Handa et al. (1992) $)^{9)}$ & $3 / \mathrm{F}$ & $\mathrm{C} 2$ & Resection & Good & At least 8 months \\
\hline Nemecek et al. $(2006)^{6)}$ & $54 / \mathrm{M}$ & $\mathrm{T} 12$ & Resection & Good & Unclarified \\
\hline van Es et al. $(2013)^{10)}$ & $62 / \mathrm{F}$ & L1 & Resection & Good & 33 days \\
\hline Johnson et al. $(2014)^{11)}$ & Teenage/- & C6 & Resection & Good & At least 13 days \\
\hline Bell et al. $(2014)^{12)}$ & $68 / \mathrm{F}$ & T5 & Resection & Unclarified & Unclarified \\
\hline *Ronchetti et al. (2015) ${ }^{1)}$ & $51 / \mathrm{F}$ & T1-4 & Resection & Good & Unclarified \\
\hline Caglar et al. $(2005)^{13)}$ & $74 / F$ & Conus & Resection & Good & Unclarified \\
\hline Takata et al. $(2015)^{14)}$ & $72 / \mathrm{F}$ & T9 & Resection & Good & Unclarified \\
\hline Cavuşoğlu et al. $(2010)^{15)}$ & $27 / F$ & C1 & Clipping & Good & Unclarified \\
\hline Geibprasert et al. $(2010)^{7)}$ & $43 / \mathrm{M}$ & $\mathrm{T} 4$ & Clipping & Good & 4 days \\
\hline Tanweer et al. (2012) & $67 / F$ & $\mathrm{~T} 10$ & Proximal embolization & Good & Unclarified \\
\hline Kim \& Choi $(2012)^{4)}$ & $52 / \mathrm{M}$ & $\mathrm{T} 7$ & Proximal embolization & Good & At least 2 days \\
\hline Shankar et al. $(2012)^{17)}$ & $72 / \mathrm{F}$ & L2 & Proximal embolization & Good & Unclarified \\
\hline Ronchetti et al. (2015) $)^{1)}$ & $68 / \mathrm{M}$ & $\mathrm{T} 1$ & Proximal embolization & Good & Unclarified \\
\hline Present case & $84 / \mathrm{M}$ & $\mathrm{T} 12$ & Resection & Good & 11 days \\
\hline
\end{tabular}

*Multiple aneurysms involving posterior spinal artery, F: female, M: male, Tx: treatment. 
when the parent artery is small, it is difficult to access the lesion. In our case, the parent artery was very small and its connection with the aneurysm was not apparent angiographically. We did not use intraoperative electrophysiological monitoring. PSAs have an abundant pial network and there had been no reports showing neurological deficit attributed to sacrificing the parent artery. However, Takata et al. recently presented a case of isolated PSA aneurysm in which motor evoked potential was deteriorated by temporary clipping of the PSA. ${ }^{14)}$

Some authors have reported success with conservative management, which has resulted in thrombosis of the aneurysm and spontaneous healing of the dissection. ${ }^{10,18)}$ Berlis et al. ${ }^{2)}$ reported a case in which spontaneous healing of an aneurysm was found when surgery was performed 26 days after the onset; they concluded that conservative management may be appropriate. In our case, intraoperative indocyanine green video-angiography did not show blood flow in the aneurysm, and pathological examination of the resected aneurysm showed it contained organized thrombus. Therefore, the aneurysm might have been in the process of healing spontaneously. Conversely, Henson et al. ${ }^{19)}$ reported a case of death that resulted from rebleeding 8 months after the original onset. Thus, it is difficult to distinguish which aneurysms truly require active treatment.

Koçak et al. ${ }^{20)}$ reported a patient who died of rebleeding within 24 hours of onset though surgery had been planned, and emphasized that treatment to prevent rebleeding should be performed as soon as possible. However, therapeutic interventions were delayed in some reports, suggesting difficulty with diagnosis. Similarly, surgical resection was performed 13 days after the onset in our case.

There are few relevant published reports. Furthermore, the publication of case reports may be influenced by a positive outcome bias, especially for cases undergoing surgery or endovascular treatment. Therefore, it is dangerous to draw conclusions about optimal therapy based on a few sporadic case reports. Our 84-year-old patient is the oldest of the 21 reported cases of isolated PSA aneurysm. Nevertheless, he tolerated surgery well and was thereafter able to participate in aggressive rehabilitation without the risk of rebleeding. Our case illustrates both the validity and efficacy of surgical treatment for ruptured isolated PSA aneurysms.

\section{Conflicts of Interest Disclosure}

The authors report no conflicts of interest concerning the materials or methods used in this study or the findings specified in this article. All authors have registered online Self-reported COI Disclosure Statement Forms through the website for JNS members. This study is not supported by any grant. The authors declare that no work resembling the enclosed article has been published or is being submitted for publication elsewhere.

\section{References}

1) Ronchetti G, Morales-Valero SF, Lanzino G, Wald JT: A cause of atypical intracranial subarachnoid hemorrhage: posterior spinal artery aneurysms. Neurocrit Care 22: 299-305, 2015

2) Berlis A, Scheufler KM, Schmahl C, Rauer S, Götz F, Schumacher M: Solitary spinal artery aneurysms as a rare source of spinal subarachnoid hemorrhage: potential etiology and treatment strategy. AJNR Am J Neuroradiol 26: 405-410, 2005

3) Rengachary SS, Duke DA, Tsai FY, Kragel PJ: Spinal arterial aneurysm: case report. Neurosurgery 33: 125-130; discussion 129-130, 1993

4) Kim HJ, Choi IS: Dissecting aneurysm of the posterior spinal artery: case report and review of the literature. Neurosurgery 71: E749-E756; discussion E756, 2012

5) Miyasaka K, Asano T, Ushikoshi S, Hida K, Koyanagi I: Vascular anatomy of the spinal cord and classification of spinal arteriovenous malformations. Interv Neuroradiol 30(6 Suppl 1): 195-198, 2000

6) Nemecek AN, Sviri G, Hevner R, Ghodke B, Britz GW: Dissecting aneurysm of the thoracic posterior spinal artery. Case illustration. J Neurosurg Spine 5: 555, 2006

7) Geibprasert S, Krings T, Apitzsch J, Reinges MHT, Nolte KW, Hans FJ: Subarachnoid hemorrhage following posterior spinal aneurysm. A case report and a review of the literature. Interv Neuroradiol 16: 183-190, 2010

8) Goto Y, Kamijyo Y, Yonekawa Y, Kikuchi H: Ruptured aneurysm of the posterior spinal artery of the upper cervical spinal cord: case report. Neurosurgery 22: 558-560, 1988

9) Handa T, Suzuki Y, Saito K, Sugita K, Patel SJ. Isolated intramedullary spinal artery aneurysm presenting with quadriplegia. Case report. J Neurosurg 77: 148-150, 1992

10) van Es AC, Brouwer PA, Willems PW: Management considerations in ruptured isolated radiculopial aneurysms. A report of two cases and literature review. Interv Neuroradiol 19: 60-66, 2013

11) Johnson J, Patel S, Saraf-Lavi E, Aziz-Sultan MA, Yavagal DR: Posterior spinal artery aneurysm rupture after 'Ecstasy' abuse. J Neurointerv Surg e23, 2014

12) Bell DL, Stapleton CJ, Terry AR, Stone JR, Ogilvy CS: Clinical presentation and treatment considerations of a ruptured posterior spinal artery pseudoaneurysm. J Clin Neurosci 21: 1273-1276, 2014

13) Caglar YS, Torun F, Pait G, Bagdatoglu C, Sancak T: Ruptured aneurysm of the posterior spinal artery of the conus medullaris. J Clin Neurosci 12: 603-605, 2005

14) Takata M, Takayama M, Yokoyama Y, Hayashi H, Kishida $\mathrm{N}$ : An isolated posterior spinal aneurysm resection in which intraoperative electrophysiological monitoring was successfully used to locate the lesion and to detect the possibility of ischemic complications: case report. Spine (Phila Pa 1976) 2015 [Epub ahead of print]

15) Cavuşoğlu H, Ozdilmaç A, Sahin Y, Aydin Y: Isolated posterior spinal artery aneurysm causing intracranial acute subarachnoidal hemorrhage. Acta Neurochir (Wien) 152: 721-724, 2010

Neurol Med Chir (Tokyo) 55, December, 2015 
16) Tanweer O, Woldenberg R, Zwany S, Setton A: Endovascular obliteration of a ruptured posterior spinal artery pseudoaneurysm. J Neurosurg Spine 17: 334-336, 2012

17) Shankar JJ, terBrugge K, Krings T: Subarachnoid hemorrhage following posterior spinal artery aneurysm rupture. Can J Neurol Sci 39: 531-532, 2012

18) Sato K, Roccatagliata L, Depuydt S, Rodesch G: Multiple aneurysms of thoracic spinal cord arteries presenting with spinal infarction and subarachnoid hemorrhage: case report and literature review. Neurosurgery 71: E1053-E1058; discussion E1058, 2012
19) Henson RA, Croft PB: Spontaneous spinal subarachnoid haemorrhage. Q J Med 25: 53-66, 1956

20) Koçak A, Ateş O, Cayli SR, Saraç K: Isolated posterior spinal artery aneurysm. Br J Neurosurg 20: 241-244, 2006

Address reprint requests to: Toshiro Katsuta, MD, Department of Neurosurgery, Fukuoka University, 7-45-1 Jonan-ku, Fukuoka 814-0133, Japan.

e-mail: tktktk010101@m3.dion.ne.jp 